RUNNING HEAD: DEATH REMINDERS, ALCOHOL, AND DAY OF THE WEEK

Mortality salience leads to greater consumption of an ostensibly alcoholic beverage on Friday vs other weekdays

\author{
Simon McCabe \\ University of Stirling \\ Bruce D. Bartholow \\ University of Missouri - Columbia
}

Accepted for publication in British Journal of Health Psychology published by Wiley. The final published version is available at: https://doi.org/10.1111/bjhp.12382 


\begin{abstract}
Objectives: We build on findings from terror management theory to examine how non-conscious mortality concerns may lead individuals to adhere to cultural meanings yoked to discrete timeperiods, in this case influencing consumption of an ostensibly alcoholic beverage.

Design: The study took the form of a 2 (death vs. uncertainty reminder) x 3 (Monday vs. Wednesday vs. Friday) between subjects lab-based quasi-experimental design.

Methods: 210 participants (age: $M=21.92$ years, $S D=5.33 ; 103$ males, 107 females) recruited from a UK university answered either an open-ended question to prime mortality or uncertainty cognition on either a Monday, Wednesday, or Friday. As part of an ostensible taste-test, they then consumed as much or as little of a purportedly alcoholic drink as they desired.
\end{abstract}

Results: Death reminders (vs. control topic) were found to result in more consumption of the beverage on a Friday, less consumption on a Monday, and no difference in consumption on a Wednesday.

Conclusions: Findings point to the flexible, time contingent nature of culture-oriented defenses against mortality concerns with potential implications for the efficacy of alcohol health warnings featuring mortality-related stimuli.

Keywords: terror management, existential psychology, weekend effect, alcohol, culture. 
Scholars have noted that the organization of time into units such as hours, days, and years does not reflect objective temporal units inherent in nature (Berger \& Luckmann, 1966). Rather, these divisions are, at least in part, culturally demarcated. The French, for example, for 12 years (1793 - 1805) during their revolution observed a ten-day week. Further, these culturally sanctioned units of time come to connote certain culturally informed meanings (Berger \& Luckmann, 1966). Such culture-specific conceptualizations of time may have behavioral implications for everyday affairs, including domains pertaining to physical health. For example, summer might be the time to get in shape for the beach; New Year's Day the time to kick a harmful habit; and cultural holidays such as St. Patrick's Day in Ireland and Día de los Muertos (the day of the dead) in Latin America being the time to consume large amounts of alcohol. Interestingly, Día de los Muertos juxtaposes plentiful reminders of mortality with plentiful alcohol use as individuals drink, in part, because it is a culturally scripted way to celebrate the dead. However, this also raises the possibility that temporal frames and the cultural scripts associated with them interact with mortality awareness to influence evaluations of alcohol. Given the anxiety-reducing effect of alcohol consumption (e.g., Sayette, 1999), it might be assumed that reminders of aversive events, such as one's own impending death, would result in a greater desire to drink alcohol regardless of the day of the week or time of year.

Alternatively, intuition might suggest death reminders would make health concerns more salient, prompting people to curb drinking in an effort to maintain health, irrespective of culturally ordained temporal scripts.

However, prior theory and research suggest the relationship between mortality concerns, cultural scripts, and alcohol use may be more nuanced. In particular, terror management theory (TMT; Greenberg, Pyszczynski, \& Solomon, 1986) posits that conscious and non-conscious awareness of mortality engages different motivational orientations aimed at buffering the threat 
associated with mortality-related cognition (Pyszczynksi, Greenberg, \& Solomon, 1999). Conscious thoughts of death are argued to instigate responses focused on removing death thoughts from focal attention. In contrast, and of particular relevance to the present studies, subtle or fleeting thoughts of mortality are argued to motivate people to live up to cultural norms and values, which confers existential security by allowing individuals to feel a part of something more enduring than the self, i.e., culture. Numerous findings fit this analysis; for example, mortality thoughts outside of focal attention increase in-group identification (Castano, 2004) and adherence to normative behavior (Jonas et al., 2008). In addition, studies derived from the terror management health model (Goldenberg \& Arndt, 2008), an application of TMT to health domains, demonstrate that health communications featuring a cultural celebrity are more effective when death thoughts are non-conscious (McCabe, Vail, Arndt, \& Goldenberg, 2014). Taken together, these studies suggest that people are more likely to be influenced by cultural factors (e.g., cultural celebrities, the in-group identity, and cultural codes for normative behaviors) when death thoughts are outside of conscious awareness.

Of course, cultural norms and values manifest in many ways. One manifestation is reflected in the way time is carved into discrete units and the culturally informed scripts and norms associated with those units (e.g., Bartlett, 1932; Rumelhart, 1980). Following TMT, if non-conscious mortality concerns motivate people to behave in accordance with cultural prescriptions, and cultural norms stipulate time periods that are associated with certain patterns of behavior, then after reminders of death (mortality salience; MS) people should bring their attitudes and behavior in line with the scripts yoked to culturally delineated temporal boundaries. Indeed, Routledge and Arndt (2003) found that after reading about a person who refuses to wear a watch or use a calendar, participants reminded of mortality rated the "temporal transgressor" more negatively than those in a control condition. Another study found that, following a MS 
induction, participants higher in personal need for structure expressed a greater desire for temporal organization in terms of more specific start and end times for an imaginary future holiday (Landau, Greenberg, \& Sullivan, 2009). Although these studies inform the existential value of culturally organized time, little research bridges the gap between this value and the implications scripts associated with cultural units of time have for health-related behaviors, such as alcohol consumption. Indeed, while prior research has indicated death reminders may spur people to consume more alcohol, these studies' effects may be driven by culturally bound contextual cues rather than temporal cues. For example, one study found that after death reminders students who were low in self-esteem consumed more alcohol at a nightclub (Wisman, Heflick, \& Goldenberg, 2015). Another study found that priming death thoughts led students on a university campus to be more inclined to purchase an alcoholic vs. a non-alcoholic beverage (Ein-Dor et al., 2014). Given previous research has found mortality reminders can spur adherence to normative codes of conduct (Jonas et al., 2008), it is possible these studies' effects were driven by what participants perceived to be contextual or environmental cues for normative behavior i.e., drinking in a nightclub or at university. Importantly for the study at hand, these studies did not explore the potential influence of temporal cues for normative conduct.

The weekend effect, referring to the tendency for people to imbibe more alcohol on Fridays and Saturdays relative to other days (e.g., Maggs, Williams, \& Lee, 2011), provides an opportunity to examine the possible impact of temporal cues. This effect is particularly pronounced for university students, whose perceived norms about the typical weekend tend to include drinking alcohol (Clapp, Shillington, \& Segars, 2000). Therefore, if the distinction between the weekend and weekdays reflects culturally-prescribed norms related to alcohol consumption, and non-conscious mortality concerns spur adherence to temporally sensitive cultural norms, then reminders of mortality should exacerbate the tendency to have more positive 
evaluations of alcohol on a Friday, but not on other weekdays.

\section{Preliminary studies}

The present research was informed by the findings of two pilot studies, described in detail in the online supplementary material. Participants in those studies were randomly assigned to MS or control conditions, and then were asked about their alcohol-related attitudes either when imagining drinking on a Monday versus a Friday (Pilot Study $1 ; N=160$ ), or on an actual Monday or Friday (Pilot Study 2; $N=81$ ). Across both studies, the self-report measures indicated attitudes towards alcohol were more positive when participants considered drinking on a Friday (vs. a Monday) after being reminded of death (vs. a control topic). Pilot study 1 was particularly informative in that more positive attitudes towards alcohol emerged on a Friday only when death thoughts were outside of (vs. in) focal awareness, informing the design of both Pilot study 2 and the main study described here.

\section{The present research}

Encouraged by the findings of the pilot studies, we next examined whether reminders of death would influence consumption of an ostensibly alcoholic beverage in a manner analogous to its influence on self-report measures of alcohol attitudes. Further, as an auxiliary measure, we aimed to clandestinely video record participants as they consumed the ostensibly alcoholic beverage to get an indication of speed of consumption, as prior research links speed of consumption with savoring (e.g., Vohs et al., 2013). We conducted the study on Mondays, Wednesdays, and Fridays. This was done to examine whether death reminders would lead to more consumption of an ostensibly alcoholic beverage on a Friday (when it is the cultural script to drink), but not on a Monday or Wednesday (when it is not) ${ }^{1}$.

\footnotetext{
${ }^{1}$ An additional reason for including Monday and Wednesday vs. Friday relates to the pilot studies (see supplemental material) which utilised only Friday vs Monday.
} 


\section{Method}

\section{Participants}

Meta-analyses of MS effect sizes were consulted to anticipate the sample sizes necessary to achieve a sufficient level of power (.80) to detect MS effects within each condition, should such effects be present. Burke et al. (2010) found an overall MS effect size of $r=.35(d=.75)$ on a broad range of studies using various worldview defense outcomes (e.g., defense of national identity; sports team affiliations; physical aggression). The power analysis (G*Power; Faul, Erdfelder, Buchner, \& Lang, 2009), assuming $d=.75$, prescribed a minimum of 12 participants per each of the six conditions, for a minimum total sample size of 72 participants. In case the current effect was smaller than that suggested by Burke et al., and to accommodate potential attrition and exclusion criteria, we decided to aim for more than triple this amount and collect data from a target $\mathrm{N}$ of 240 participants.

A recruitment notice was posted on a UK university portal and posters placed around campus; both students and staff were eligible ${ }^{2}$. The notice advertised a 'taste-test' study and noted the study lasted approximately 30 minutes with participants being entered into a raffle to win one of three $£ 50$ amazon gift vouchers (per semester). Sessions lasted 30 minutes and were available Mondays, Wednesdays, and Fridays between $1 \mathrm{pm}-8 \mathrm{pm}$. Two hundred and forty participants were recruited between July 2016 and September 2017. Twenty-four participants indicated they do not drink alcohol, and therefore did not complete the study. Data from six additional participants were not included in the analyses because, during post-study probing, they indicated they suspected the beverage was not alcoholic. These participants did not vary by condition, $\chi^{2}(5, \mathrm{~N}=240)=1.08, p=.96$. This left 210 participants (age: $M=21.92$ years, $S D=$

\footnotetext{
${ }^{2}$ Note that the pilot studies were conducted using US samples. We do not believe that this is an issue given the weekend effect has been demonstrated in the US (e.g., Maggs et al., 2011), and in the UK and Europe (e.g., Sieri et al., 2003).
} 
5.33; 103 males, 107 females), with 35 participants within each of the six conditions being randomly assigned to receive the mortality salience (vs. control) task via Qualtrics software (Qualtrics, Provo, UT). Assignment to day of the week was handled via email scheduling and was based on the participants' availability. In cases participants were available multiple days (193 participants of the final 210), we randomized to condition. As such, there was constrained random assignment to condition for this variable.

\section{Materials and procedure}

\section{Premeasures and mortality salience manipulation.}

Personality measure. As a premeasure and to bolster believability in the cover story, participants first completed a short measure of personality (ten-item personality index; Gosling, Rentfrow, \& Swann, 2003). Example items include, "I see myself as extraverted, enthusiastic" and "I see myself as conventional, uncreative." Responses were made using a scale anchored at 1 (Disagree strongly) and 7 (Agree strongly). After completing this measure, participants were randomly assigned to the MS or control condition.

Mortality salience. As in numerous previous studies (see Burke, Martens, \& Faucher, 2010), those in the MS condition responded to two open-ended questions presented to the participant as a recently designed way to assess personality: "What do you think will happen to you when you die," and "What do you think happens to you as you physically die." Following previous research (e.g., Arndt et al., 2009), those assigned to the control condition responded to parallel questions about uncertainty - "What do you think happens when you experience uncertainty," and "What do you think happens to you physically when you feel uncertain".

Distraction task. Participants then completed a set of filler tasks designed to distract them from conscious thoughts of mortality. They first completed the expanded positive $(\alpha=.79)$ and negative $(\alpha=.81)$ affect schedule (PANAS-X; Watson \& Clark, 1991). This 60-item scale 
included instructions to "indicate to what extent you feel this way right now," and included emotions such as "cheerful", "sad", and "hostile", anchored at 1 (very slightly) and 5

(extremely). Participants then read a one-page extract from a short story (Camus' [1958], The Growing Stone), rated its descriptiveness, and indicated the perceived gender of the author. Previous research in which these tasks have been used to distract participants from death thoughts suggests completing them takes approximately 5-8 minutes (see Martens, Burke, Schimel, \& Faucher, 2011). Prior research also indicates this distraction task is an effective method for allowing mortality cognition to be removed from focal awareness (see Steinman \& Updegraff, 2015, for a meta-analysis).

Taste test instructions. After completing the above measures and clicking onto the next screen, participants saw some instructions that read, "You will now taste a beverage. You have been selected to try a beer. Please open the container in front of you, open the beer with the bottle opener, and pour it into the plastic cup provided. Once you have done this, you may begin drinking the beer. Drink as much or as little as you like. Once you are done, please click the next button and answer the questions about the taste of the beer. Do not drink any more beer once you have pressed the next button".

Demographics and probe questions. Participants then completed a measure assessing demographic information including age, sex, nationality, race, political orientation, and religious affiliation (please see Table 1 in supplementary materials for demographic data by condition). We included two probe questions: "what do you think this study is about?", and "had you heard about terror management theory before this study?" (yes/no). Upon completing the study, participants were briefly interviewed by the researcher and answered some probe questions to check for suspicion before receiving a verbal debriefing explaining the true purpose of the study. Finally, participants completed the form to be entered into the $£ 50$ raffle. 
Study procedure. Upon arrival, participants were greeted by a researcher who delivered a cover story consistent with how the study was advertised. Participants were run one at a time and were informed that they were to take part in a 'taste test' study exploring the link between personality and preference for different kinds of food and drink. The cover story led the participant to believe the team were examining all kinds of consumables including alcohol, and that the participant had been randomly selected to consume alcohol. Participants who agreed to continue completed the informed consent form and the researcher recorded the participant's date of birth from a valid ID to ensure they were of legal drinking age. The researcher then informed the participant that the study had three parts: the first part involving some personality measures (which also, unbeknownst to the participants, included the mortality salience manipulation presented as an 'innovative personality measure'), the second part involving a taste test, and the final part involving some questions related to the beverage. Participants were reminded of this three-part structure via a welcome screen presented on a tablet, which served to administer instructions, manipulations, and measures (using Qualtrics -- Provo, UT). Participants were informed that the survey would tell them when to open a manila envelope that was placed on the desk close to where they were seated. The envelope contained a $275 \mathrm{ml}$ bottle of non-alcoholic beer (Becks blue, with all labels removed and branded cap taped over), a transparent plastic cup, and a bottle opener. When the participants reached the taste test portion of the study, they were instructed via the tablet to open the envelope and pour as much or as little of the beer into the plastic cup as they wished ${ }^{3}$. Participants were told they could take as much or as little time as

\footnotetext{
${ }^{3}$ The reason for pouring the beer into the transparent cup was that we had originally aimed to attain a measure of how fast participants were drinking, number of gulps/sips, and overall time spent drinking via a hidden camera located in a picture frame featuring an image of some flowers. However, after running 19 participants and examining the footage we identified that the video quality was not sufficient to determine when the participant had finished drinking and if there was any liquid left in the cup. Further, participants would often move the empty manila envelope in such a way that it blocked the view of the camera and there were technical issues involving the image suddenly blacking out or the recording ending abruptly. Due to these issues we removed this element from future sessions and do not discuss it further.
} 
they wanted before advancing to the final portion of the study. Once done with the 'taste test' phase, participants proceeded to answer some self-report exploratory questions evaluating the beverage (see supplementary materials). Once participant had finished the study there was a debriefing session and the participant then left the room. The remaining liquid from the bottle and cup was then poured into a graduated cylinder to assess the amount (ml) of liquid remaining, which was subtracted from the total $275 \mathrm{ml}$ to give an index of volume consumed.

\section{Analyses}

\section{Primary analyses}

Volume of beer consumed. Consumed volumes were submitted to a 2 (MS; death vs. uncertainty) x 3 (Study day; Monday vs. Wednesday vs. Friday) between-subjects analysis of variance $(\mathrm{ANOVA})^{4}$.

\section{$\underline{\text { Secondary analyses }}$}

Affect. To examine potential affective consequences of the MS manipulation, day of the week and potential interactions, the two higher-order scales of the PANAS-X were submitted to separate 2 (MS) x 3 (Study day) factorial ANOVAs

Gender influence. Gender differences on consumption were examined via a 2 (MS vs uncertainty) x 3 (Monday vs. Wednesday vs. Friday) x 2 (Male vs. Female) ANOVA in a set of exploratory analyses.

\section{Results}

Volume of beer consumed. The ANOVA revealed a main effect of study day, $F(2,204)$ $=7.15, p=.001, \eta_{\mathrm{p}}^{2}=.07$. Simple effect comparisons showed that less beer was consumed on Monday vs. Wednesday, $t(138)=-2.58, p=.010, d=-0.439$, and more beer was consumed on

\footnotetext{
${ }^{4}$ To account for the inflated experimentwise type 1 error rate given number of multiple comparisons, all pairwise ttests reported are Bonferroni corrected.
} 
Friday vs. Monday, $t(138)=3.68, p<.001, d=.626$, but not on Friday vs. Wednesday, $t(138)=$ $1.09, p=.274, d=.185$.

The analysis also produced an interaction between MS and study day, $F(2,204)=5.39, p$ $=.005, \eta_{\mathrm{p}}^{2}=.05$ (see Table 1$)$. Within the MS condition, less beer was consumed on Monday vs. Wednesday, $t(68)=-2.82, p=.005, d=-.684$, and more beer was consumed on Friday vs. Monday, $t(68)=4.92, p<.001, d=1.193$, and on Friday vs. Wednesday, $t(68)=2.09, p=.037, d$ $=.506$. There were no differences in beer consumption across study days in the uncertainty condition, all $t \mathrm{~s}<.833, p \mathrm{~s}>.406$. Tests of the effect of MS (vs. uncertainty) within each study day showed that people consumed less beer after MS (vs. uncertainty) on Mondays, $t(68)=$ 2.47, $p=.014, d=-.599$, and more beer after MS (vs. uncertainty) on Fridays, $t(68)=2.15, p=$ $.032, d=.521$, with no significant difference in consumption after MS (vs. uncertainty) on Wednesdays, $t(68)=-.48, p=.629, d=-.116$.

Affect. The ANOVA examining positive affect showed a main effect of study day, $F(2,204)=4.46, p=.013, \eta^{2} \mathrm{p}=.02$, such that positive affect was greater on Friday vs. Monday, $t(68)=2.46, p=.015, d=.596$, and greater on Friday vs. Wednesday, $t(68)=2.69, p=.008, d=$ .652 , with no significant difference on Wednesday vs. Monday, $t(68)=-2.4, p=.814, d=-.582$. There were no other main or interaction effects on positive or negative affect, ps $>.111$.

Gender influence. The ANOVA revealed a significant main effect of gender for the volume of beer consumed, $F(1,198)=9.50, p=.002, \eta^{2} \mathrm{p}=.05$, such that males drank more than females. No other main or interactive effects involving gender emerged on any of the other dependent measures, $p s>.174$. The relationships from the primary analysis were unchanged when gender was included in the model.

\section{General Discussion}

The present research provides evidence that mortality concerns can motivate adherence to 
norms yoked to culturally stipulated time units in ways that may have implications for healthrelated attitudes and behaviors, here specifically concerning alcohol. Building from two pilot studies demonstrating more positive attitudes towards alcohol after a death reminder when imagining it was a Friday (vs. Monday) and on an actual Friday or Monday, we found these effects translated to actual consumption. These findings offer both basic and applied contributions, with implications for alcohol attitudes and health messages as well as for understanding the nature of cultural norms about time and existential motivation.

Previous research demonstrates that cultural norms help direct psychological reactions to mortality awareness. For example, integrating the focus theory of normative conduct (Cialdini, Reno, \& Kallgreen, 1991) with TMT, Jonas and colleagues (2008) found that salient norms (e.g., about prosocial behavior) lead to norm-consistent behavior following MS. The current work extends these findings by showing that temporally bound norms about engaging in a healthrelated behavior (drinking) also may be exacerbated by MS. The present studies thus offer novel contributions to understanding the interface between cultural norms for behavior yoked to discrete temporal units and management of existential concerns.

Indeed, the influence of typical associations with days of the week expands prior considerations of how existential concerns contribute to the temporal life in which people live. Extending studies on preferences for temporal structure and reactions to those who eschew temporal conventions (Landau et al., 2009; Routledge \& Arndt, 2003), the present studies document subtler influences of death-related thought on conforming to cultural conventions of time. Rather than explicitly asking participants about attitudes toward time (or priming people with particular norms, as in Jonas et al., 2008), the present study relied on participants' natural associations with particular weekdays (i.e., the weekend effect) and examined their ostensible alcohol consumption on a Monday, Wednesday, or Friday. This was sufficient to reveal how 
cultural associations with conceptions of time pervade peoples' everyday life and can be engaged by reminders of mortality.

Regarding limitations, the study did not include true randomization to the day of the week factor. We were only able to randomize in the cases where participants were available multiple days of the week to complete the study. While none of the demographic measures that we included varied by condition, it is possible that some unmeasured characteristics were more prevalent in samples on one day vs. another. Future studies might attempt to replicate the results reported here with true randomization to the day of the week factor. The laboratory setting of the study also likely limits the ecological validity of the findings. People rarely-if ever-consume alcohol in settings similar to a university psychological laboratory, which lacks most of the contextual features common to typical drinking environments (e.g., those found in a pub or one's home). As such, testing the model presented here across more ecologically valid contexts is a reasonable next step before any firm recommendations for policy or interventions can be made. Nevertheless, we tentatively raise the following issues about approaches to curbing excessive alcohol consumption, particularly among university students. Given the numerous negative consequences associated with university student binge drinking (e.g., Foster, Caravelis, \& Kopak, 2014; Hingson et al., 2002), identifying the social psychological antecedents of risky alcohol use is critical. Recent research has identified a few ways that the management of deathrelated cognition may be involved in this form of risky behavior. For example, studies indicate that non-conscious death-thoughts increase the likelihood of alcohol consumption (Ein-Dor et al., 2014), and that such cognition may be of particular concern for exacerbating risky alcohol consumption when people base their self-worth on drinking (Jessop \& Wade, 2008; Wong \& Dunn, 2013), or have low self-esteem (Wisman, Heflick, \& Goldenberg, 2015). The present study offers the novel insight that it is not just bases of self-worth or low self-esteem that can 
predispose people to such effects, but operative cultural norms as well - in this context those tethered to cultural associations with temporal demarcations. More concretely, potential interventions might take the form of altering materials that feature persuasive attempts to curb alcohol consumption, based on day of the week. A public house or bar might, for example, utilize warning that feature the threat of death on Monday through Thursday, but switch to other approaches (e.g., threat of embarrassment or financial loss) that do not feature death during the weekend period.

This study also extends prior research by informing how it is not only contextual cues that can inform scripts for behavior after MS, but also temporal ones. Prior studies found, for example, alcohol consumption was greater after MS in contexts where participants had been at a nightclub (Wisman, Heflick, \& Goldneberg, 2015) or were in the social area of a university campus (Ein-Dor et al., 2014). The present study was conducted in a lab, presumably lacking environmental cues to drink (beyond the taste-test cover story - which was kept constant across conditions), with the day of the week being examined as the temporal cue and finding an influence on drinking contingent on weekday. This raises an interesting direction for future research to consider, i.e., the reach and degree of influence of temporal vs. contextual factors in spurring adherence to normative behaviors after MS. This in turn also raises potential implications for public health communications and suggests another reason caution might be warranted in the use of mortality-related themes in public service announcements, particularly on a Friday (vs. other days of the week). Relatedly, it is interesting to note that there was a decrease in amount drank on a Monday vs. Wednesday or Friday after MS (vs. control). This may suggest that not only can MS spur adherence to engaging in greater alcohol consumption, but also contribute to lower consumption contingent on what the script is yoked to the temporal unit. While further research is needed, one tentative hypothesis is that Monday connotes a cultural 
script not to drink alcohol. Again, this leads to a consideration of how health warnings featuring mortality-related stimuli might both undermine and bolster the effectiveness of such warnings contingent on the currently activated temporal unit and scripts for alcohol use associated with it. In conclusion, alcohol attitudes and consumption were shown to be impacted by cultural defenses against mortality concerns that were expressed differently depending on the current time period (i.e., Monday vs. Wednesday vs. Friday), arguably because of the meanings associated with such periods. The findings presented are tentative, but also generative both theoretically for understanding the interface between existential threat, time, and cultural norms, and practically for considering temporal aspects of health promotions or deterrents that feature mortality reminders. 


\section{References}

Arndt, J., Vess, M., Cox, C. R., Goldenberg, J. L., \& Lagle, S. (2009). The psychosocial effect of thoughts of personal mortality on cardiac risk assessment. Medical Decision Making, 29, 175-181. doi:10.1177/0272989X08323300

Bartlett, F.C. (1932). Remembering: A Study in Experimental and Social Psychology. Cambridge, England: Cambridge University Press

Berger, P. L., \& Luckmann, T. (1991). The social construction of reality: A treatise in the sociology of knowledge. Penguin UK.

Burke, B. L., Martens, A., \& Faucher, E. H. (2010). Two decades of terror management theory: A meta-analysis of mortality salience research. Personality and Social Psychology Review, 14, 155-195. doi:10.1177/1088868309352321

Camus, A. (1958). The Growing Stone. In Albert Camus: Exile and the Kingdom. New York: Knopf.

Castano, E. (2004). In case of death, cling to the ingroup. European Journal of Social Psychology, 34, 375-384. doi:10.1002/ejsp.211

Cialdini, R. B., Reno, R. R., \& Kallgreen, C. A. (1990). A focus theory of normative conduct: Recycling the concept of norms to reduce littering in public places. Journal of Personality and Social Psychology, 58, 1015-1026. doi:10.1016/S0065-2601(08)60330-5

Clapp, J. D., Shillington, A. M., \& Segars, L. B. (2000). Deconstructing contexts of binge drinking among college students. The American Journal of Drug and Alcohol Abuse, 26, 139-154. doi:10.1081/ADA-100100596

Ein-Dor, T., Hirschberger, G., Perry, A., Levin, N., Cohen, R., Horesh, H., \& Rothschild, E. (2014). Implicit Death Primes Increase Alcohol Consumption. Health Psychology, 33, 748-751. doi:10.1037/a0033880 
Faul, F., Erdfelder, E., Lang, A. G., \& Buchner, A. (2007). G* Power 3: A flexible statistical power analysis program for the social, behavioral, and biomedical sciences. Behavior Research Methods, 39, 175-191. doi:10.3758/bf03193146

Foster, C., Caravelis, C., \& Kopak, A. (2014). National College Health Assessment Measuring Negative Alcohol-Related Consequences among College Students. American Journal of Public Health Research, 2, 1-5. doi: 10.12691/ajphr-2-1-1

Goldenberg, J.L., \& Arndt, J. (2008). The implications of death for health: A terror management health model for behavioral health promotion. Psychological Review, 115, 1032-1053. doi:10.1037/a0013326

Gosling, S. D., Rentfrow, P. J., \& Swann, W. B., Jr. (2003). A very brief measure of the Big-Five personality domains. Journal of Research in Personality, 37, 504-528. doi:10.1016/S0092-6566(03)00046-1

Greenberg, J., Pyszczynski, T., \& Solomon, S. (1986). The causes and consequences of a need for self-esteem: a terror management theory. In R. F. Baumeister (Ed.), Public self and private self(pp.189-212). New York: Springer-Verlag. doi:10.1007/978-1-4613-95645_10

Hingson, R. W., Heeren, T., Zakocs, R. C., Kopstein, A., \& Wechsler, H. (2002). Magnitude of alcohol-related mortality and morbidity among US college students ages 18-24. Journal of Studies on Alcohol, 63, 136-144. doi:10.15288/jsa.2002.63.136

Jessop, D. C., \& Wade, J. (2008). Fear appeals and binge drinking: A terror management theory perspective. British Journal of Health Psychology, 13, 773-788.

doi:10.1348/135910707X272790

Jonas, E., Martens, A., Niesta, D., Fritsche, I., Sullivan, D., \& Greenberg, J. (2008). Focus theory of normative conduct and terror management theory: The interactive impact of mortality 
salience and norm salience on social judgment. Journal of Personality and Social Psychology, 95, 1239-1251. doi:10.1037/a0013593

Landau, M. J., Greenberg, J., \& Sullivan, D. (2009). Defending a coherent autobiography: When past events appear incoherent, mortality salience prompts compensatory bolstering of the past's significance and the future's orderliness. Personality and Social Psychology Bulletin, 35, 1012-1020. doi: 10.1177/0146167209336608

Maggs, J. L., Williams, L. R., \& Lee, C. M. (2011). Ups and downs of alcohol use among firstyear college students: Number of drinks, heavy drinking, and stumble and pass out drinking days. Addictive Behaviors, 36, 197-202. doi:10.1016/j.addbeh.2010.10.005

Martens, A., Burke, B.L., Schimel, J., and Faucher. E.H. (2011) Same but different: metaanalytically examining the uniqueness of mortality salience effects. European Journal of Social Psychology 41(1): 6-10. doi:10.1002/ejsp.767

McCabe, S., Vail, K.E.III, Arndt, J., \& Goldenberg, J.L. (2014). Hails from the crypt: A terror management health model investigation of health and celebrity endorsements. Personality and Social Psychology Bulletin, 40, 289-300. doi:10.1177/0146167213510745

Pyszczynski, T., Greenberg, J., \& Solomon, S. (1999). A dual-process model of defense against conscious and unconscious death-related thoughts: an extension of terror management theory. Psychological review, 106, 835-845. doi:10.1037/a0039729

Routledge, C., \& Arndt, J. (2003). The terror of time: Mortality salience and time-related worldviews. Manuscript in preparation.

Rumelhart, D.E.(1980) Schemata: the building blocks of cognition. In: R.J. Spiro et al. (Eds) Theoretical Issues in Reading Comprehension, Hillsdale, NJ: Lawrence Erlbaum. Sayette, M. A. (1999). Does drinking reduce stress? Alcohol Research and Health, 23, 250-255. 
Sieri, S., Agudo, A., Kesse, E., Klipstein-Grobusch, K., San-Jose, B., Welch, A. A., ... \& Tjønneland, A. (2002). Patterns of alcohol consumption in 10 European countries participating in the European Prospective Investigation into Cancer and Nutrition (EPIC) project. Public Health Nutrition, 5, 1287-1296.

Steinman, C. T., \& Updegraff, J. A. (2015). Delay and death-thought accessibility: A metaanalysis. Personality and Social Psychology Bulletin, 41, 1682-1696.

Vohs, K. D., Wang, Y., Gino, F., \& Norton, M. I. (2013). Rituals enhance consumption. Psychological Science, 24, 1714-1721. doi:10.1177/0956797613478949

Watson, D., \& Clark, L. A. (1991). The PANAS-X: Preliminary manual for the positive and negative affect schedule - Expanded form. Unpublished manuscript, Southern Methodist University, Dallas, TX, USA.

Wisman, A., Heflick, N., \& Goldenberg, J. L. (2015). The great escape: The role of self-esteem and self-related cognition in terror management. Journal of Experimental Social Psychology, 60, 121-132. doi:10.1016/j.jesp.2015.05.006

Wong, N. C., \& Dunn, S. S. (2013). Binge Drinking and TMT: Evaluating Responses to AntiBinge Drinking PSAs from a Terror Management Theory Perspective. Studies in Media and Communication, 1, 81-95. doi:10.11114/smc.v1i2.216 